\title{
Immune surveillance of tumors
}

\author{
Jeremy B. Swann and Mark J. Smyth \\ Cancer Immunology Program, Peter MacCallum Cancer Centre, East Melbourne, Victoria, Australia, and \\ Department of Pathology, University of Melbourne, Parkville, Victoria, Australia.
}

\begin{abstract}
The ability of the immune system to identify and destroy nascent tumors, and to thereby function as a primary defense against cancer, has been debated for many decades. Recent findings by a number of investigators in both mouse models of cancer and humans with cancer now offer compelling evidence that particular immune cell types, effector molecules, and pathways can sometimes collectively function as extrinsic tumor suppressor mechanisms. This work provides the basis for further study of natural immunity to cancer and for rational use of this information in the design of immunotherapies in combination with other conventional cancer treatments.
\end{abstract}

\section{Introduction}

The immune system has three primary roles in the prevention of tumors. First, the immune system can protect the host from virus-induced tumors by eliminating or suppressing viral infections. Second, the timely elimination of pathogens and prompt resolution of inflammation can prevent the establishment of an inflammatory environment conducive to tumorigenesis. Third, the immune system can specifically identify and eliminate tumor cells on the basis of their expression of tumor-specific antigens or molecules induced by cellular stress.

The third process is referred to as tumor immune surveillance, whereby the immune system identifies cancerous and/or precancerous cells and eliminates them before they can cause harm. The idea that the immune system, which so effectively protects the host from microbial pathogens, might also recognize and destroy tumor cells was first discussed over a century ago and has recently been reviewed in detail (1). Despite tumor immune surveillance, tumors do develop in the presence of a functioning immune system, and therefore the updated concept of tumor immunoediting (1) is a more complete explanation for the role of the immune system in tumor development.

The tumor immunoediting concept is divided into 3 phases, designated elimination, equilibrium, and escape (2) (Figure 1). The elimination phase of cancer immunoediting is exactly the same process described in the initial theory of tumor immune surveillance, whereby the immune system detects and eliminates tumor cells that have developed as a result of failed intrinsic tumor suppressor mechanisms. The elimination phase can be complete, when all tumor cells are cleared, or incomplete, when only a portion of tumor cells are eliminated. In the case of partial tumor elimination, the theory of immunoediting is that a temporary state of equilibrium can then develop between the immune system and the developing tumor. During this period it is envisaged that tumor cells either remain dormant or continue to evolve, accumulating further changes (such as DNA mutations or changes in gene expression) that can modulate the tumor-specific antigens and stress-induced

Nonstandard abbreviations used: DMBA, 7,12-di-methylbenz[a]-anthracene; IL-12R, IL-12 receptor; iMC, immature myeloid cell; MCA, methylcholanthrene; MGUS, monoclonal gammopathy of undetermined significance; $\mathrm{MM}$, multiple myeloma; MSI, microsatellite instability; MSI-H, high MSI; NKG2D, NK group 2, member D; PCD, paraneoplastic cerebellar degeneration; Tag, SV40 large T antigen; TIL, tumor-infiltrating lymphocyte; TPA, 12-O-tetradecanoyl-phorbol-13-acetate; TRAIL, TNF-related apoptosis-inducing ligand.

Conflict of interest: The authors have declared that no conflict of interest exists. Citation for this article: J. Clin. Invest. 117:1137-1146 (2007). doi:10.1172/JCI31405 antigens that they express. As this process continues, the immune system exerts a selective pressure by eliminating susceptible tumor clones where possible. The pressure exerted by the immune system during this phase is sufficient to control tumor progression, but eventually, if the immune response still fails to completely eliminate the tumor, the process results in the selection of tumor cell variants that are able to resist, avoid, or suppress the antitumor immune response, leading to the escape phase. During the escape phase the immune system is no longer able to contain tumor growth, and a progressively growing tumor results.

This new concept of cancer immunoediting is a more satisfactory interpretation of various clinical and experimental data, which implicate a protective role for the immune system in tumor control. The observations that have led to the concept of tumor immunoediting are reviewed here, with a particular focus on experimental data from various mouse models of cancer and clinical experience.

\section{Evidence for cancer immunoediting in animal models of cancer}

Spontaneous tumor development in immunodeficient mice. The simplest approach for testing the role of the immune system in controlling tumor development is to remove specific components of the mouse immune system and monitor the mice for the development of tumors. Predominantly through the use of gene-targeted mice, this approach has demonstrated that a number of immune effector cells and pathways are important for suppression of tumor development (Table 1).

A role for the adaptive immune system in suppressing tumor growth was revealed when it was shown that 129/Sv RAG2-deficient mice, which lack both B and T cells, develop spontaneous adenocarcinoma of the intestine and lung (35\% and $15 \%$, respectively, of all mice analyzed) at $15-16$ months of age, and an additional $50 \%$ of mice develop intestinal adenomas (3). Interestingly, when Rag2 $2^{-/}$mice were also deficient for STAT1, an important mediator of signaling induced by both type I and type II IFN, tumor incidence is further increased, and the spectrum of tumors broadens to include breast adenocarcinomas ( $\sim 40 \%$ of mice), colon adenocarcinomas $(-10 \%$ of mice), or both ( $20 \%$ of mice) (3). Together, these results suggest that both the innate and the adaptive arms of the immune system are involved in the prevention of tumors, as mice lacking both IFN signaling and an adaptive immune system develop a broader spectrum of tumors than mice only lacking an adaptive immune system.

Mice lacking $\mathrm{T}$ cell and NK cell cytotoxic effector pathways have also been shown to develop spontaneous tumors. For example, mice that lack perforin, a cytotoxic molecule used by cytotoxic cells 


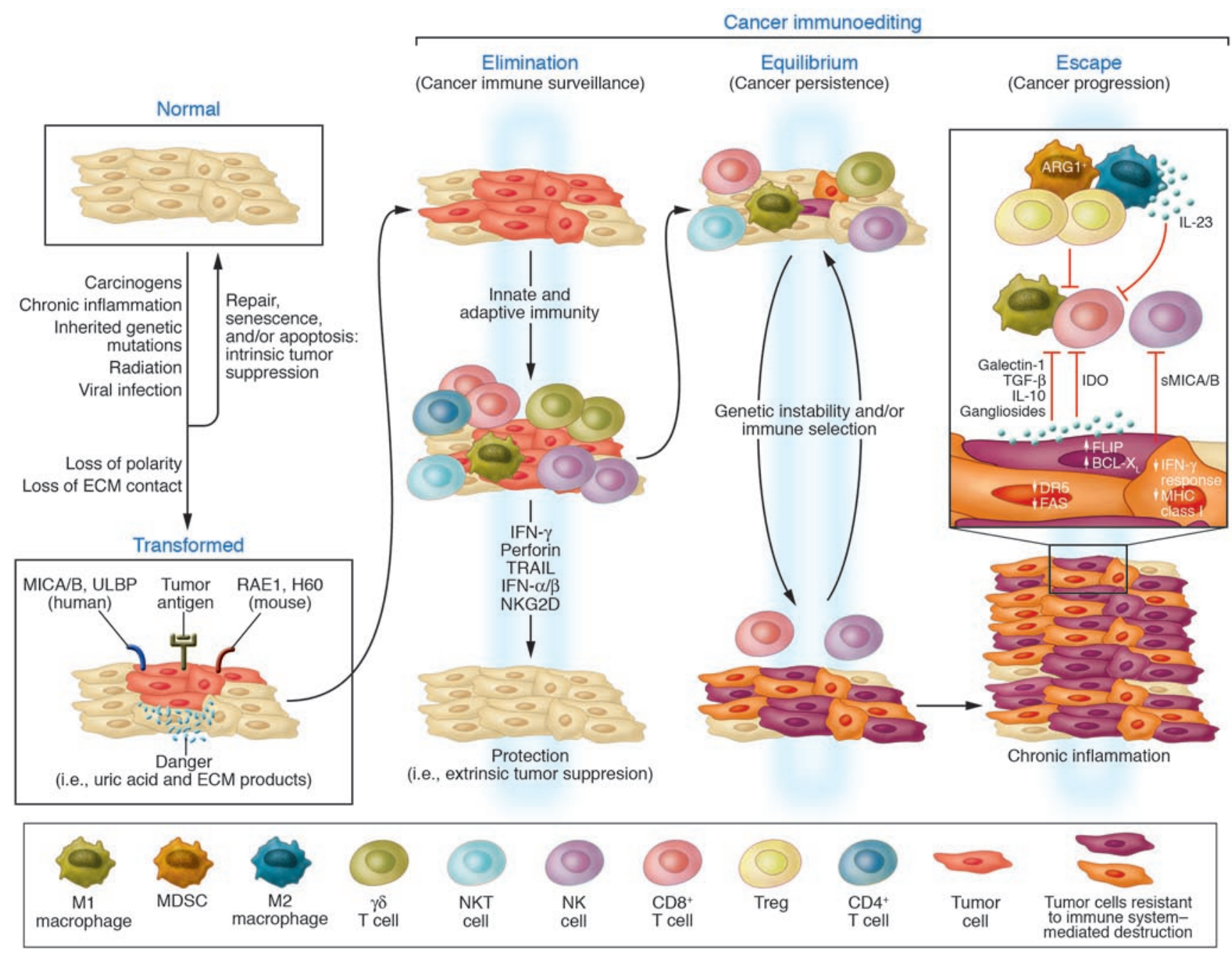

Figure 1

Extrinsic tumor suppression by the immune system. Transformed cells escaping intrinsic control are subjected to extrinsic tumor suppressor mechanisms that detect and eliminate developing tumors before they become clinically apparent. This is known as the elimination phase of a broader process that has been termed cancer immunoediting. Cancer immunoediting takes into account the observation that the immune system both protects the host against tumor development and promotes tumor growth. Cancer immunoediting is now considered a process composed of 3 phases: elimination, or cancer immune surveillance; equilibrium, a phase of tumor dormancy where tumor cells and immunity enter into a dynamic equilibrium that keeps tumor expansion in check; and escape, where tumor cells emerge that either display reduced immunogenicities or engage a large number of possible immunosuppressive mechanisms to attenuate antitumor immune responses leading to the appearance of progressively growing tumors. These phases have been termed the $3 \mathrm{Es}$ of cancer immunoediting. DR5, death receptor 5 ; IDO, indoleamine 2,3-dioxygenase; MICA/B, MHC class I chain-related antigens A and B; RAE1, retinoic acid early transcript 1; sMICA/B, soluble MICA/B; ULBP, UL16-binding protein. Figure adapted with permission from Advances in Immunology (117).

such as $\mathrm{CD} 8^{+} \mathrm{T}$ cells and NK cells to form membrane pores in target cells, develop lymphomas with age. These spontaneous lymphomas are of B cell origin, develop in older mice (>1 year of age) regardless of the mouse strain $(4,5)$, and, when transplanted into WT mice, are rejected by $\mathrm{CD}^{+} \mathrm{T}$ cells (4). B cell lymphomas also arise in mice lacking both perforin and $\beta_{2} \mathrm{~m}$, and tumor onset is earlier and occurs with increased prevalence compared with mice lacking only perforin. In addition, B cell lymphomas derived from mice lacking both perforin and $\beta_{2} \mathrm{~m}$ are rejected by either NK cells or $\gamma \delta \mathrm{T}$ cells following transplantation to WT mice, rather than by $\mathrm{CD}^{+} \mathrm{T}$ cells (as in tumors derived from mice lacking only perforin), demonstrating that cell surface expression of MHC class I molecules by tumor cells can be an important factor in determining which effector cells mediate immune protective effects (6). Intriguingly, mutations in the gene encoding perforin have also been identified in a subset of lymphoma patients (7), although it is not clear whether this contributes to disease. Mice lacking the death-inducing molecule TNFrelated apoptosis-inducing ligand (TRAIL) or expressing a defective mutant form of the death-inducing molecule FASL have also been shown to be susceptible to spontaneous lymphomas that develop with late onset $(8,9)$. These aging studies have clearly demonstrated a critical role for cytotoxic pathways in immunoregulation and/or immunosuppression of spontaneous tumor development in mice.

Several cytokine-deficient mice also develop spontaneous malignancies $(5,10,11)$. In one study, approximately $50 \%$ of IFN- $\gamma$-deficient C57BL/ 6 mice were found to develop $\mathrm{T}$ cell lymphomas that 


\section{Table 1}

Immunodeficient mouse strains that develop spontaneous tumors

\begin{tabular}{|c|c|c|c|}
\hline Strain & Description & Phenotype & Reference \\
\hline SCID & Lack $T$ and $B$ cells & Mice develop T cell lymphomas ( $15 \%$ of mice) & 118 \\
\hline $\operatorname{Rag}^{-/-}$ & Lack $T$ and $B$ cells & $\begin{array}{l}\text { Mice develop intestinal adenomas }(\sim 50 \%) \text {, or adenocarcinoma } \\
\text { of the intestine }(\sim 35 \%) \text { or lung }(\sim 15 \%) \text {; tumors detected at } \\
15-16 \text { months of age }\end{array}$ & 3 \\
\hline Rag2-/-Stat1-/- & $\begin{array}{l}\text { Lack T and B cells, } \\
\text { deficient for type I and II IFN signaling }\end{array}$ & $\begin{array}{l}\text { Mice develop intestinal adenomas like } \operatorname{Rag}^{--} \text {mice }(\sim 20 \%) \text {, but also } \\
\text { develop adenocarcinoma of the breast }(\sim 40 \%) \text {, colon }(\sim 10 \%) \text {, or } \\
\text { breast and colon }(\sim 20 \%) \text {; tumors detected at } 12-18 \text { months of age }\end{array}$ & 3 \\
\hline Perforin ${ }^{--}$ & Lack perforin & Mice develop B cell lymphomas at 14-21 months of age & 4 \\
\hline lfng ${ }^{--}$ & Lack IFN- $\gamma$ & $\begin{array}{l}\text { Mice develop lymphomas (predominantly T cell) at } 13-19 \text { months } \\
\text { of age; effect is strain dependent (C57BL/6 are susceptible, } \\
\text { BALB/c are resistant) }\end{array}$ & 5 \\
\hline Perforin'-1fng-/- & Lack both perforin and IFN- $\gamma$ & $\begin{array}{l}\text { Mice develop B cell lymphomas similar to those observed in perforin- } \\
\text { deficient mice, but with earlier onset and increased frequency }\end{array}$ & 5 \\
\hline Perforin'--B2m-- & Lack both perforin and MHC class I expression & $\begin{array}{l}\text { Mice develop B cell lymphomas similar to those observed in perforin- } \\
\text { deficient mice, but with earlier onset and increased frequency }\end{array}$ & 6 \\
\hline Lmp2 $2^{--}$ & Defective MHC class I antigen presentation & Mice develop uterine neoplasms (36\%) by 12 months of age & 119 \\
\hline Trait-- & Lack TRAIL & About $25 \%$ of mice develop lymphomas late in life (>400 days) & 8 \\
\hline Gmcsf--Ifng ${ }^{-1}$ & Lack GM-CSF and IFN- $\gamma$ & $\begin{array}{l}\text { Mice develop a range of malignancies, including lymphomas and } \\
\text { solid tumors (predominantly ovarian choriocarcinoma, luteomas, } \\
\text { or teratomas) }\end{array}$ & 11 \\
\hline $1 / 12 r b 2^{-1-}$ & Lack IL-12R $\beta 2$ & Mice develop plasmacytomas, lung carcinomas, or both (50\%) & 10 \\
\hline
\end{tabular}

are predominantly disseminated lymphomas, although some cases of thymic lymphoma are also noted; interestingly, the susceptibility of $\mathrm{Ifng}^{-/-}$mice to T cell lymphomas is strain dependent (5). Furthermore, the spectrum of tumors observed in IFN- $\gamma$ - and STAT1-deficient mice do not overlap, despite STAT1 being a crucial signaling molecule downstream of the IFN- $\gamma$ receptor, indicating either that these molecules have some nonoverlapping activities or that the background strain has a modifying influence on tumor type. In addition, C57BL/ 6 mice lacking both IFN- $\gamma$ and perforin display accelerated B cell lymphoma onset compared with perforin-deficient mice (5), indicating that IFN- $\gamma$ has an important role in modifying the progression to $\mathrm{B}$ cell lymphoma in perforin-deficient mice. IL-12 and IL-18 are important IFN- $\gamma$-inducing cytokines; however, studies of aging have demonstrated that neither IL-12- nor IL-18-deficient mice display increased incidence of tumor development compared with WT mice (5).

A possible link between tumor immunity and autoimmune or infection-induced inflammation has been raised by several studies. Curiously, with age, $50 \%$ of mice lacking the $\beta 2$ subunit of the IL-12 receptor (IL-12R $\beta 2$ ) develop plasmacytomas or lung carcinoma in the context of the autoimmune disease immune complex mesangial glomerulonephritis (10). It is presently unclear why IL-12-deficient mice on the same genetic background as the IL-12R $\beta 2$-deficient mice do not display either autoimmunity or spontaneous tumor development. Furthermore, mice deficient for both IFN- $\gamma$ and GM-CSF have also been found to develop tumors with age; in this case, tumor development is associated with acute or chronic inflammatory lesions in a range of organs, and maintaining mice on the antibiotic enrofloxacin prevents (or at least delays) tumor onset (11). Collectively, these findings demonstrate that the immune system can suppress tumor development, but they do not constitute proof for tumor immunoediting per se. The finding that antibiotic treatment could prevent tumor development in $\mathrm{Gm}_{\text {-csf }}{ }^{/-} \mathrm{Ifn} \mathrm{g}^{-1}$ mice raises the possibility that rather than directly eliminating tumor cells, the immune system might prevent tumor growth by the timely elimination of infections, thereby limiting inflammation, which is known to facilitate tumor development (12). However, this finding cannot

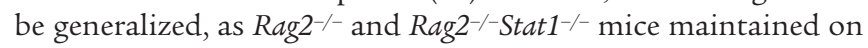
the same antibiotics and housed under strict specific pathogen-free conditions still display heightened tumor incidence despite testing negative for common pathogens with known links to malignancy and showing no signs of idiopathic inflammation (3).

Carcinogen-induced tumors in immunodeficient mice. In order to more readily study the role of the immune system in shaping tumorigenesis, researchers have turned to inducible tumor models, including carcinogen-induced tumors. The 2 most commonly employed carcinogen-induced tumor models are fibrosarcomas induced using methylcholanthrene (MCA) and skin papillomas induced by a combination of 7,12-di-methylbenz[a]-anthracene (DMBA) and 12-O-tetradecanoyl-phorbol-13-acetate (TPA). To date, a number of mice with defined immunodeficiencies have been tested for their susceptibility to carcinogens (Table 2).

Rag2 ${ }^{-/-}$and SCID mice, both of which lack an adaptive immune system, display a heightened susceptibility to tumor induction with MCA $(3,13)$, and similar findings have been made in nude mice (14), which lack most T cells. Both $\alpha \beta$ and $\gamma \delta$ T cells were subsequently found to be important in suppressing MCA-induced tumors, as mice deficient for either of these $\mathrm{T}$ cell subsets display increased susceptibility to tumor induction (15). Interestingly, $40 \%$ of tumors derived from Rag $2^{-/-}$mice are rejected when transplanted into WT recipients but grow progressively in either Rag2/hosts or mice depleted of $\mathrm{CD}^{+}$and $\mathrm{CD}^{+} \mathrm{T}$ cells, whereas tumors derived from WT mice grow readily when transplanted into either WT or Rag2-/- hosts (3). These observations are important because they not only show that carcinogen-induced tumors arise more frequently in immunodeficient mice, but also that tumors derived from these immunodeficient mice are more immunogenic than those arising in mice with a functional immune system. CD1d- 


\section{Table 2}

Carcinogen-induced tumors in immunodeficient mice

$\begin{array}{ll}\begin{array}{l}\text { Strain } \\ \text { Rag2 }\end{array} & \begin{array}{l}\text { Description } \\ \text { Lack T and B cells }\end{array} \\ \text { Nude } & \text { Lack most T cells due to lack of thymus }\end{array}$

\begin{tabular}{|c|c|}
\hline SCID & Lack $T$ and $B$ cells \\
\hline $\mathrm{Tcrb}^{-/-}$ & Lack $\alpha \beta$ T cells \\
\hline Tcrd $^{-1-}$ & Lack $\gamma \delta$ T cells \\
\hline$C d 1 d^{--}$ & Lack CD1d-restricted T cells \\
\hline Ja18-/ & Lack semi-invariant NKT cells \\
\hline RAE1 transgenic & Defective killing through the NKG2D pathway \\
\hline Perforin ${ }^{--}$ & Lack perforin \\
\hline Trait/- & Lack TRAIL \\
\hline Ifngrt-- & Lack IFN- $\gamma$ R1 \\
\hline lfng $^{-/}$ & Lack IFN- $\gamma$ \\
\hline Stat1-/- & Deficient for type I and type II IFN signaling \\
\hline Perforin ${ }^{-1}$ Ifng $^{-1-}$ & Lack both perforin and IFN- $\gamma$ \\
\hline $1 /-12 p 35^{-/-}$ & Lack IL-12 \\
\hline $1 /-23 p 19^{-/}$ & Lack IL-23 \\
\hline $1 /-12 p 40^{-/}$ & Lack IL-12 and IL-23 \\
\hline Ifnar1-1- & Lack type I IFN signalling \\
\hline $\mathrm{Cd} 80^{-/} \mathrm{Cd} 86^{-/-}$ & Lack costimulatory molecules CD80 and CD86 \\
\hline
\end{tabular}

\section{Phenotype}

Increased susceptibility to MCA-induced sarcomas, about $40 \%$ of sarcomas rejected following transplantation into WT recipients Increased susceptibility to MCA-induced sarcomas, but dependent on strain; compared with sarcomas derived from WT mice, sarcomas from nude mice express higher levels of MHC class I and grow less aggressively when transplanted into WT hosts Increased susceptibility to MCA-induced sarcomas Increased susceptibility to MCA-induced sarcomas Increased susceptibility to MCA-induced sarcomas and DMBA/TPA-induced skin carcinogenesis

Increased susceptibility to MCA-induced sarcomas, some sarcomas rejected on transplantation into WT recipients

Increased susceptibility to MCA-induced sarcomas, some sarcomas rejected on transplantation into WT recipients

Increased susceptibility to DMBA/TPA-induced skin carcinogenesis Increased susceptibility to MCA-induced sarcomas Increased susceptibility to MCA-induced sarcomas Increased susceptibility to MCA-induced sarcomas, reconstituting sarcomas with IFNGR1 facilitates their rejection by WT mice

Increased susceptibility to MCA-induced sarcomas and $\mathrm{N}$-methyl-N-nitrosourea-induced lymphomas Increased susceptibility to MCA-induced sarcomas Increased susceptibility to MCA-induced sarcomas Increased susceptibility to MCA-induced sarcomas and $\mathrm{N}$-methyl-N-nitrosourea-induced lymphomas

Decreased susceptibility to DMBA/TPA-induced skin carcinogenesis Increased susceptibility to MCA-induced sarcomas, decreased susceptibility to DMBA/TPA-induced skin carcinogenesis Increased susceptibility to MCA-induced sarcomas Increased susceptibility to UV-induced skin carcinogenesis
Reference

3

14,120

13

15

15

16

16,17

19,121 restricted $\mathrm{T}$ cells, which bridge the innate and adaptive arms of the immune system, also have a role in suppressing MCA-induced fibrosarcomas. Mice that lack the potential TCR component J 18 are unable to generate the semi-invariant V $\alpha 14-J \alpha 18$-containing TCR expressed by NKT cells and show increased susceptibility to fibrosarcoma induction $(16,17)$. Interestingly, similar to immunogenic tumors from $\operatorname{Rag}^{-/-}$mice, a portion of tumors arising in mice lacking J $\alpha 18$ are rejected when transplanted into WT hosts (18), indicating that they are immunogenic and that the absence of NKT cells might contribute to the enhanced tumor development in Rag2-/- mice. Evidence that the innate immune system is also important in suppression of MCA fibrosarcomas was provided in mice chronically depleted of NK cells, since these mice display increased tumor incidence (16).

To further understand how the immune system suppresses fibrosarcoma growth, a number of mice deficient for specific immune effector molecules and pathways have been examined, including mice lacking perforin (19), TRAIL (20), IL-12 (16), IFN- $\gamma(21)$, IFNAR1 (a component of the type I IFN receptor) (22), and NK group 2, member D (NKG2D) (23). Each of these mouse strains demonstrates enhanced susceptibility to fibrosarcoma induction, suggesting that cytotoxic cells (NK cells and CD8 ${ }^{+} \mathrm{CTLs}$ ) use these pathways to suppress tumor growth in vivo. Interestingly, further investigation of this phenomenon revealed that tumor cells are important targets for the antitumor effects of IFN- $\gamma(21)$, whereas the host hematopoietic system is the target of the antitumor effects of type I IFN (22), suggesting that the ability of type I IFNs to induce antitumor activity in immune cells might be the critical mode of action for this cytokine family.

This interpretation of the results regarding the role of IFN- $\gamma$ in prevention of MCA-induced fibrosarcomas has not been universally accepted, however, with other researchers proposing that IFN- $\gamma$ contributes to an inflammatory response that results in the encapsulation of injected MCA (a process referred to as a foreign body reaction), limiting its spread and thereby reducing its carcinogenic effects (24). However, the finding that $\mathrm{Ifn}^{-/-}$mice are more susceptible to lymphomas induced by the soluble carcinogen N-methyl-N-nitrosourea (25), where encapsulation of the carcinogen is not possible, is at odds with this concept. The finding that restoration of IFN- $\gamma$ receptor 1 (IFN- $\gamma \mathrm{R} 1$ ) expression in MCA-induced tumor lines from Ifngr $1^{-/-}$mice leads to a delay in tumor growth or complete tumor rejection when such tumors are transplanted into WT mice also strongly suggests IFN- $\gamma$ is not merely a driver for encapsulation of MCA.

A role for the immune system in regulating the development of DMBA/TPA-induced papillomas has also been investigated (Table 2). With the DMBA/TPA model, skin carcinomas are induced by the topical application of DMBA (the tumor initiator), followed by repetitive doses of TPA (the tumor promoter). In this model, lesions progress from benign papillomas through to metastatic squamous cell carcinomas, and the number and progression of the lesions is dependent on the mouse strain. While $\gamma \delta \mathrm{T}$ cells confer protec- 


\section{Table 3}

Genetic mouse tumor models and immunodeficiency

\begin{tabular}{|c|c|}
\hline Strain & Description \\
\hline $\begin{array}{l}p 53^{--1} \text { Ifngr1-1-; } 129 / \mathrm{Sv} \text { or } \\
129 / \mathrm{Sv} \times \text { C57BL/6 background }\end{array}$ & Lack p53 and IFN- $\gamma$ R \\
\hline$p 53^{+-}$Tap $1^{-1-}$ & Heterozygous for p53 and lack TAP1 \\
\hline$p 53^{+/-} L m p 2^{-/-}$ & Heterozygous for p53 and lack LMP2 \\
\hline p53 $3^{+-}$Perforin $-/$ & Heterozygous for p53 and lack perforin \\
\hline p53 $3^{+-}$Trait/- & Heterozygous for p53 and lack TRAIL \\
\hline Trait/- HER2/neu transgenic & $\begin{array}{l}\text { Lack TRAIL and transgenic for } \\
\text { HER2/neu (under MMTV promoter) }\end{array}$ \\
\hline$D r 5^{-/-} A p c^{M i n /+}$ & $\begin{array}{l}\text { Lack TRAIL receptor DR5; } \\
\text { heterozygous for } A p c^{M i n}\end{array}$ \\
\hline$p 53^{--}$Dr5-- & Lack p53 and DR5 \\
\hline Ifng $^{-1-H T L V-T a x ~ t r a n s g e n i c ~}$ & $\begin{array}{l}\text { Lack IFN- } \gamma \text { and transgenic for human } \\
\text { HTLV type I-derived Tax } \\
\text { (under granzyme B promoter) }\end{array}$ \\
\hline
\end{tabular}

\section{Phenotype}

Earlier tumor onset, broader spectrum of tumors compared with $p 53^{--/}$; no change versus $p 53^{--}$

No change in tumor onset or spectrum versus $p 53^{+/-T a p 1^{+/+}}$

No change in tumor onset or spectrum versus $p 53^{+-} L m p 2^{+/+}$

Accelerated onset of B cell lymphoma

Increased tumor incidence

No change in mammary tumor onset, volume, or survival of host

No change in colon adenocarcinoma onset

No change in tumor onset or spectrum

Accelerated leukemia and/or lymphoma onset
Reference

21,24

124

124

4

8

8

125

33

Apc Min/+, mutant multiple intestinal neoplasia (Min) allele of adenomatosis polyposis coli.

tion from DMBA/TPA-induced papillomas (15), $\alpha \beta$ T cells seem to promote tumor progression in this model of carcinogenesis (26). One mechanism by which $\gamma \delta \mathrm{T}$ cells might regulate tumor development is through NKG2D recognition of the stress ligand retinoic acid early transcript 1 (RAE1), expression of which is induced in the skin by DMBA/TPA treatment. NKG2D-expressing dendritic epidermal $\gamma \delta \mathrm{T}$ cells can kill RAE1-expressing targets in vitro (15), but in transgenic mice expressing RAE1 in the skin, NKG2D expression is downmodulated on lymphocytes and consequently these mice are more susceptible to papilloma induction than are WT mice (27). Collectively these data indicate that the NKG2D pathway is important in the control of carcinogen-induced tumors. IL-23 and IL-12 are functionally related heterodimeric cytokines that both contain the IL-12 $\beta$ subunit (although paired with distinct subunits) and activate distinct receptors that each contain the IL-12R $\beta 1$ subunit. Recently, Langowski et al. induced papillomas in mice that lack either the IL-23-specific subunit that pairs with IL-12 $\beta$ or the IL-12specific subunit that pairs with IL-12 $\beta$ (28). Interestingly, mice that lack functional IL-23 are resistant to tumor development, whereas mice that lack functional IL-12 develop increased numbers of papillomas compared with WT mice. In a broad panel of human tumors, the authors also found substantial upregulation of the mRNAs encoding both subunits of IL-23 and hypothesized that expression of IL-23 in human tumors has a causative role in promoting tumor development. Although the mechanism by which IL-23 promotes tumor growth requires further clarification, it has been found that carcinogen-treated IL-23-deficient mice produce less IL-17 (a cytokine with tumor growth-promoting activity; refs. 29, 30) than do WT controls. Moreover, since the DMBA/TPA model of cancer is known to be dependent on a strong inflammatory response, more work is needed to explore the relative importance of inflammation versus immunoediting in other primary tumor models and whether these are distinct or overlapping processes. Tumors induced by physical carcinogens such as UV radiation also seem to be controlled by the immune system (31), and it is thought that UV-induced immune suppression is an important factor in the development of UV-induced tumors, as UV-induced tumors are often immunogenic when transplanted into naive hosts but grow in immunosuppressed recipients or recipients depleted of $\mathrm{CD}^{+} \mathrm{T}$ cells (32).
Genetic tumor models in immunodeficient mice. Data supporting the ability of the immune system to suppress tumor development in genetic models of mouse cancer is less extensive. Several studies have demonstrated that the immune system can protect mice from malignancy induced by genetic means, whereas others have shown no effect (Table 3). Of the genetic tumor models, mice heterozygous for the tumor suppressor p53 $\left(p 53^{+/-}\right.$mice $)$have provided the most convincing evidence for tumor immune surveillance, indicating a role for perforin (4), TRAIL (8), and IFN- $\gamma$ R1 (21) in immune control of tumor development. Transgenic expression of oncogenes under the control of tissue-specific promoters has become a common technique to investigate the process of tumorigenesis; however, few of these studies have addressed the role of the immune system in delaying or preventing the development of tumors. A role for IFN- $\gamma$ in suppressing tumor development has been observed in mice expressing the human T cell leukemia virus (HTLV) type 1-derived oncogene Tax under the control of a granzyme B promoter (HTLV-Tax transgenic mice); these mice develop a lymphoproliferative disease similar to human adult T cell leukemia/lymphoma. When HTLV-Tax mice are crossed with Ifng-/- mice, tumor onset is accelerated, suggesting that IFN- $\gamma$ has a protective effect in this model (33). In a second transgenic tumor model, using the oncogene encoding the SV40 large T antigen (Tag), IFN- $\gamma$ deficiency has no effect on tumor onset or the spectrum of tumors that develop (34); instead, tumor growth causes the skewing of $\mathrm{T}$ cell responses, with tumor-specific $\mathrm{T}$ cells producing TGF- $\beta$ rather than IFN- $\gamma$. Similarly, $\mathrm{CD}^{+} \mathrm{T}$ cells have no effect on tumor development in the rat insulin promoterTag4 (RIP-Tag4) pancreatic tumor model, where Tag is expressed in the pancreas under the control of RIP, resulting in the development of pancreatic tumors (35). These results again confirm that the role of the immune system in suppressing tumors is likely to be dependent on the natural history of the individual tumor and the oncogenic changes that it has acquired.

Summary of mouse models. In summary, various cell types, including $\alpha \beta$ and $\gamma \delta$ T cells, NKT cells, and NK cells, have been implicated in the processes of elimination and immunoediting, along with a number of effector molecules, including perforin and TRAIL, and cytokines, including IFN- $\gamma$, type I IFNs, and IL-12. It is important to note that the effector cells and cytokines thought to be involved in elimination 
and immunoediting differ among models, demonstrating that the success of immunoediting and the evidence of its occurrence varies among experimental systems. Indeed, there are models in which the immune system seems to have little influence on the rate of tumor onset or progression (34) and models in which the immune system has a distinct protective role, such as the carcinogen-induced tumor models outlined above. The level of immune regulation and tolerance (a state of nonresponsiveness to specific antigens) imparted by the tumors in each of these models might explain, at least in part, why in some cases the effect of subtracting immune elements on tumor progression is less overt. Blocking these tolerance mechanisms might reveal the true mechanisms of tumor suppressor immunity. Therefore, to date, observations in mouse models are an indirect readout of tumor elimination, and it has not yet been possible to observe the establishment and subsequent immune-mediated regression (i.e., elimination) of an autochthonous tumor.

Evidence for the equilibrium phase of tumor immunoediting, during which the tumor and antitumor immune response coevolve, is least advanced and again indirect. MCA-induced fibrosarcomas from mice lacking IFN- $\gamma$, IFNAR1, TRAIL, or T cells as well as lymphomas from mice lacking perforin can be eliminated when transplanted into immunocompetent recipients but grow progressively when transplanted into mice of the same genotype as that in which they arose. These data suggest that the immune system sculpts the immunogenic profile of evolving tumors, the exact process thought to occur during the equilibrium phase of immunoediting. In contrast to the equilibrium phase, abundant evidence exists for the escape phase of immunoediting (see below), and the growth of tumors in immunocompetent hosts is the most basic evidence that tumors can escape immune control. More specific evidence for the escape phase comes from studies in which an abortive, concurrent antitumor immune response is detected in mice with progressive tumor growth.

\section{Evidence for cancer immunoediting in humans}

A number of clinical observations have provided evidence supporting the notion of tumor immune surveillance in humans. The increased risk of tumor development in immunosuppressed patients, instances of spontaneous tumor regression, and the appearance of tumorreactive $T$ cells and $B$ cells in relation to improved prognosis all point to a role for the immune system in suppressing tumor growth.

Immunosuppressed patients. Immunosuppression (caused by either primary or secondary immunodeficiencies) and therapy to prevent transplant rejection are often associated with a heightened risk of malignancy. Immunosuppression to prevent transplant rejection is clearly associated with a heightened risk (3- to 100-fold increase) of developing certain types of malignancy (36). These diseases are predominantly lymphomas; however, a range of solid tumors with no known viral association also occur with increased frequency $(36,37)$. In addition to these tumors in patients receiving immunosuppressive drugs, a number of tumors (especially lymphomas) also occur commonly in patients with primary and acquired immunodeficiencies; however, these are generally thought to have a viral etiology (37). Perhaps a more convincing argument that cancer immunoediting does not occur in individuals who are immunosuppressed would be if preexisting, dormant tumors grew after immunosuppression commenced or if an existing tumor regressed following withdrawal of immunosuppression. No studies have yet addressed these issues in depth. In summary, although evidence from immunosuppressed patients supports the theory of immunoediting, further investigation in this area is warranted, as the contributions of viral infection, cytotoxic drugs, and persistent inflammation to tumorigenesis in this setting must also be taken into consideration.

Microsatellite unstable human tumors. Some tumor types exhibit a particular type of genetic instability referred to as microsatellite instability (MSI), where defects in DNA mismatch repair mechanisms lead to the duplication or deletion of short repeated sequences of DNA known as microsatellites. Immunoediting seems to have an important role in controlling genetically unstable colorectal tumors in particular $(38,39)$. The infiltration of colorectal cancer cell nests by $\mathrm{CD}^{+} \mathrm{T}$ cells is associated with a favorable prognosis (40), and this association is further strengthened in cases where tumors exhibit high levels of MSI (MSI-H) (38, 39, 41). Strikingly, MSI-H tumors are often strongly infiltrated with lymphocytes, including activated $\mathrm{CD}^{+} \mathrm{T}$ cells (41), and lymphoid follicles are often present (39), indicative of a potent local immune response. The high rate of mutation in MSI-H tumors has been shown to result in the generation of a number of novel tumor antigens that can be recognized by B cells (42), CD4 ${ }^{+} \mathrm{T}$ cells (43), and $\mathrm{CD} 8^{+} \mathrm{T}$ cells (42). Together, these findings suggest that the generation of antigenic peptides as a result of genomic instability might result in the priming of a protective $\mathrm{CD}^{+} \mathrm{T}$ cell-mediated immune response in patients with MSI-H colorectal cancers. Interestingly, this finding might not be unique to colorectal cancers, as gastric medullary cancers, which frequently exhibit MSI-H (41.2\% of cases), are more prominently infiltrated with lymphocytes than are nonmedullary gastric cancers, which rarely display a MSI-H phenotype (1.6\% of cases) (44). This trend has also been observed in a study of resectable pancreatic cancer (45) and in patients with non-Hodgkin lymphoma (46).

Human tumor-infiltrating lymphocytes. Tumor infiltration by $\mathrm{T}$ cells, NK cells, or NKT cells has been associated with an improved prognosis for a number of different tumor types (2, 47-49). However, tumor infiltration by some leukocytes, such as macrophages and Tregs, can have a detrimental impact on tumor progression. An association between favorable patient prognosis and tumor-infiltrating lymphocytes (TILs) was first observed in patients with melanoma $(47,48)$, where it was shown that patients with high levels of $\mathrm{CD}^{+} \mathrm{T}$ cell infiltration survive longer than those whose tumors contain low numbers of lymphocytes. Since then, various melanoma-specific antigens have been identified, and the presence of melanoma-specific $T$ cells recognizing a range of different antigens has been confirmed in various studies (reviewed in ref. 49). Taken together, these studies indicate that melanoma TILs are often tumor specific but respond to a wide range of different antigens. However, it is important to note that the presence of melanomaspecific CTLs in the blood is not predictive of survival, although the presence of such cells among TILs can be an indicator of enhanced survival in patients undergoing immunotherapy (50).

The plasma cell malignancy multiple myeloma (MM) might also be a system in which immunoediting can be evaluated in a clinical setting (51). The advantage of studying MM is that several stages of disease have been identified, and disease progresses from a premalignant state, known as monoclonal gammopathy of undetermined significance (MGUS), through to terminal disease (52). The ability to detect this premalignant phase of disease allows for immunologic monitoring to assess the contribution of the immune system to preventing and/or inhibiting progression to MM (53). Intriguingly, such monitoring has revealed that $\mathrm{T}$ cells derived from the bone marrow of patients with MGUS mount strong responses to autologous premalignant cells; these responses are not detected in patients with MM (53). These findings are consistent with a $\mathrm{T}$ cell response holding pre- 
malignant cells in check (i.e., equilibrium) followed by the eventual failure of this response to control such abnormal plasma cell clones, resulting in the eventual transition to MM (i.e., escape).

Paraneoplastic autoimmune syndromes. In some cases, patients with tumors experience disease symptoms that are caused by the presence of a tumor but are not the result of local tumor growth. These diseases are referred to as paraneoplastic syndromes; if caused by activation of an immune response specific for self antigens expressed on tumor cells, they are known as paraneoplastic autoimmune syndromes. Paraneoplastic autoimmune syndromes may be caused by cross-reactivity between the antitumor immune response and neurologic antigens, and the onset of neurologic symptoms often precedes diagnosis of a previously undetected tumor. Interestingly, certain paraneoplastic autoimmune syndromes are associated with particular tumor types and particular autoantibodies (54), and the fine specificity of the antineuronal antibodies found in patients is often related to the type of tumor present $(55,56)$. For example, paraneoplastic cerebellar degeneration (PCD) is a neurologic syndrome that arises in some patients with gynecologic malignancies and Hodgkin disease; these patients typically exhibit high titers of Yo-specific autoantibodies (in the case of gynecologic tumors) or thioredoxin reductase 1-specific autoantibodies (in the case of Hodgkin disease) (57). Not only autoantibodies, but CTLs specific for antigens shared by tumors and Purkinje cells have been detected in the blood of PCD patients (58), and tumors from patients with PCD often show prominent infiltration with lymphocytes and plasma cells, which is indicative of a local immune response at the tumor site. In most cases, PCD is terminal, and the only patients that generally survive this condition are those that achieve complete tumor remission in response to therapy, once again demonstrating that the tumor is the probable driver of the immune response that is both self and tumor reactive. Interestingly, PCD symptoms can precede tumor diagnosis by a number of years (59), indicating that antitumor responses might be primed even by undetectable, microscopic tumors early in their evolution. It remains to be determined whether the antitumor immune response substantially delays tumor growth in patients with PCD, and such analysis is likely to be confounded by the lethality of the disease as a result of neurologic complications.

Summary of human cancer immunoediting. As is the case for mouse studies, varying evidence has been gathered supporting each of the distinct phases of human tumor immunoediting. The phenomenon of spontaneously regressing melanoma lesions accompanied by the clonal expansion of $\mathrm{T}$ cells is presently the strongest evidence for the elimination phase of cancer immunoediting in humans (60-62). Generally speaking, however, cases of spontaneous tumor regression are rare, and it is presently unknown why regression is most common in melanoma. Spontaneous tumor regression accompanied by lymphocyte infiltration has also been noted for a number of other tumor types, including basal cell carcinoma (63), malignant lymphoma (64), Merkel cell carcinoma (65), mesothelioma (66), lung carcinoma (67), and congenital fibrosarcoma (68); however, the role of the lymphocyte infiltrate in tumor regression has not been established in these cases, and doing so is likely to be difficult due to the rarity with which such cases are observed.

Clinical evidence supporting the existence of the equilibrium phase of immunoediting is provided by a number of findings $(53,69,70-75)$. First, the existence of an immune response to premalignant MGUS cells that eventually progress to MM is consistent with the equilibrium phase, with the immune system controlling, but not eliminating, MGUS cells that eventually evolve and progress to malignancy (53). Passive immunization with idiotypespecific antibody, in conjunction with either cytokine therapy or chemotherapy, can induce remission in some patients with lowgrade B cell lymphoma; however, tumor cells are not completely eliminated and can be detected in the blood or bone marrow for up to 8 years following clinical remission (69). A role for the immune system in establishing long-term remission has also been suggested by studies of pediatric acute myeloid leukemia patients treated with either chemotherapy or chemotherapy combined with autologous bone marrow transplantation (70).

Clinical evidence suggests that tumors can remain dormant in patients for many years, and cases of relapse after long periods (at least 10 years) of tumor remission have been noted (71-73), making immune control with subsequent escape an intriguing possibility in these cases. In a similar vein, cases of transmission of tumors from organ donor to recipient have also been noted $(74,75)$. In such cases, it is possible that the tumor was being held under control by an immunologic mechanism in the donor and that transplantation of the organ into an immunosuppressed host allowed tumor outgrowth. Once again, although this is consistent with the theory of tumor immunoediting, other explanations for the growth of donor-derived malignancies are possible, such as alterations in tumor oncogene expression from a common cancer stem cell.

The escape phase is the best characterized of the three in both mice and humans. For example, the growth of melanomas clearly results in the priming of a tumor-specific immune response, even though this response is often insufficient to completely eliminate tumors (76). Similarly, the antitumor immune response seen in patients with paraneoplastic autoimmune syndromes indicates that an ongoing immune response is, in many cases, insufficient to control tumor growth, even when it is sufficient to destroy normal self tissues. How tumors escape immune control is now an active area of research, and the number of escape mechanisms reported is ever increasing.

\section{Failure of cancer immunoediting - tumor escape}

There is a considerable amount now known about tumor escape. In some cases it seems that the immune system is incapable of influencing tumor progression, but in many cases it seems that although the immune system prevents or delays tumor growth, it is eventually overwhelmed or evaded, and the tumor progresses. For example, tumors driven by expression of Tag develop in immunocompetent mice, even though the tumors express an antigen that is readily recognized by the immune system (34). Despite the inability of the immune system to control the growth of Tag-induced tumors, a Tagspecific immune response is often primed; however, this response declines, is overwhelmed, or is evaded, and tumors eventually progress. The failure of the immune system to control tumor growth in Tag-transgenic mice can occur at a number of levels, including the induction of central or peripheral tolerance. Central tolerance refers to the process by which self-reactive $T$ cells are deleted or converted to a regulatory phenotype in the thymus (77). Clearly, however, central tolerance fails to eliminate all tumor- and/or self-reactive T cells, as is evident by the presence of tumor-specific $T$ cells that recognize nonmutated self antigens (such as those belonging to the cancer/ testis antigen family) in cancer patients (49).

In cases where tumor-associated antigens are not expressed in the thymus, or when $\mathrm{T}$ cells specific for tumor antigens escape central tolerance, tumor growth can induce $\mathrm{T}$ cell tolerance in the periphery. Peripheral tolerance can occur through a deletional mechanism, the induction of antigen unresponsiveness, or the skewing of 
$\mathrm{T}$ cell functional responses, and evidence for each of these mechanisms has been observed in tumor models $(34,78)$. Often, immunotherapies that are able to induce protective responses when given before tumor challenge/onset (that is, prophylactically) fail against established tumors $(35,79-81)$. Importantly, in a number of cases, although tumor-specific immunity is compromised in tumor-burdened mice, there is often no generalized immune deficiency (82), indicating that tumors can specifically dampen down the induction of effective antitumor immunity. Concomitant immunity is one example where tumor growth clearly primes an antitumor immune response that ultimately fails to control the tumor's growth: a mouse challenged with a given tumor will reject a subsequent challenge with the same tumor at a distant site, despite the fact that the initial tumor continues to grow (83-85). All of these examples collectively demonstrate that, in addition to central and peripheral tolerance, failure of antitumor immunity can be the result of factors within the local tumor environment. Similar findings have also been noted in clinical studies (86), and the lack of appropriate stimulation in the tumor microenvironment might cause the ineffective responses generated by TILs (87).

Many factors in the tumor microenvironment have been shown to contribute to tumor escape, including activation of T cells in the absence of appropriate costimulation, resulting in anergy (88); expression of T cell-inhibitory molecules such as B7-H1 (89), HLA-G (90), and HLA-E (91) by tumor cells; tumor antigen loss or downregulation of $\mathrm{MHC}$ molecules (92); the presence of both $\mathrm{CD} 4^{+} \mathrm{CD} 25^{+}$Tregs and CD1d-restricted $T$ cells that suppress antitumor immunity (93); soluble suppressive factors expressed by tumors, such as TGF- $\beta$ (94-96), VEGF (97), IL-10 (98), and gangliosides (99); expression of indoleamine 2,3-dioxygenase (IDO) in tumors (reviewed in ref. 100); tumor resistance to cytotoxic pathways, as observed in tumors with mutations in the gene encoding FAS $(101,102)$, mutations in the gene encoding the TRAIL receptor death receptor 5 (DR5) (103), and overexpression of the antiapoptotic molecules FLIP and BCL- $\mathrm{X}_{\mathrm{L}}$ $(104,105)$; and expansion of immunosuppressive myeloid cell populations $(106,107)$. In the latter case, expansion of immature myeloid cell populations has been observed in a number of studies and is associated with profound suppression of $\mathrm{T}$ cell responses in both mice and humans $(108,109)$. Several tumor-derived cytokines have now been implicated in the expansion of immature myeloid cells (iMCs), including VEGF (110), IL-1 $\beta$ (111), and GM-CSF (112). The mechanism of iMC suppression is complex and seems to involve contributions from either iNOS (also known as NOS2) or arginase 1 (ARG1) (113-115). The signaling pathways activated by these molecules enable iMCs to inhibit $\mathrm{T}$ cell responses in various ways, including induction of apoptosis, inhibition of proliferation, or induction of a regulatory phenotype. Type 2 macrophages found at tumor sites have also been implicated in suppression of tumor immunity and seem to share some functional properties with iMCs (116). From these examples it can clearly be seen that by generating the appropriate environment, tumors can skew the immune response such that tumor growth, rather than elimination, is favored.

\section{Future directions}

We are armed with a lot of knowledge from mouse models of cancer regarding the various roles played by the immune system in tumor development; however, immunodeficient humans clearly have a far greater susceptibility to lethal viruses and pathogens than do immunocompromised mice in specific pathogen-free mouse facilities, and therefore the opportunities for observing increased spontaneous tumor formation in patients with mutations in specific genes encoding immune effector molecules are few. On the positive side, we might expect to see a wealth of new human genetic information generated by emerging high-throughput sequencing techniques, and this might enable a very rapid assessment of polymorphisms and mutations in immune pathways of large cancer patient cohorts that will confirm or deny the importance of key immune pathways in controlling human tumor development. The value of early detection, predicted risk, and prevention cannot be underestimated in this context.

It is also important in the future to clarify which particular immune cells are prognostic for each distinct type of cancer. Gene expression profiling and proteomics are going to have a key role in defining the major positive and negative immune indicators of human cancer progression. More also needs to be understood about the mechanisms required for the induction of a therapeutically relevant immune response in humans in the face of tumor escape. Indeed, even in mice, there is very little information about the extensive and continual coexistence of tumor cells, host stromal cell populations, and infiltrating leukocytes during any particular stage of tumor development. It is therefore important for tumor immunologists to gather a clearer molecular definition of the tumor microenvironment in real time, and perhaps only advanced imaging technologies will allow the specific cellular relationships to be properly discerned.

Immunotherapy is potentially synergistic with other treatment modalities, and approaches that trigger tumor cell death, alter the tumor microenvironment, reduce tolerogenic mechanisms, and stimulate immune responses might act in concert. An improved understanding of the immunobiology of cancer immunoediting and a molecular definition of how tumors are shaped by this process will undoubtedly bring us closer to a more effective use of immunotherapy together with other conventional cancer treatments to prevent, control, and/or eradicate established cancer.

\section{Acknowledgments}

M.J. Smyth was supported by a National Health and Medical Research Council of Australia (NH\&MRC) Research Fellowship and Program Grant. J.B. Swann was supported by an Australian Postgraduate Research Scholarship. The authors would like to thank Robert Schreiber for helpful discussion.

Address correspondence to: Mark J. Smyth, Cancer Immunology Program, Peter MacCallum Cancer Centre, Locked Bag 1, A'Beckett Street, East Melbourne 8006, Victoria, Australia. Phone: 613-9656-3728; Fax: 613-9656-1411; E-mail: mark.smyth@petermac.org.

\footnotetext{
1. Dunn, G.P., Bruce, A.T., Ikeda, H., Old, L.J., and Schreiber, R.D. 2002. Cancer immunoediting: from immunosurveillance to tumor escape. Nat. Immunol. 3:991-998.

2. Dunn, G.P., Old, L.J., and Schreiber, R.D. 2004. The three Es of cancer immunoediting. Annu. Rev. Immunol. 22:329-360.

3. Shankaran, V., et al. 2001. IFNgamma and lymphocytes prevent primary tumour development and
}

shape tumour immunogenicity. Proc. Natl. Acad. Sci.U.S. A. 10:1107-1111.

4. Smyth, M.J., et al. 2000. Perforin-mediated cytotoxicity is critical for surveillance of spontaneous lymphoma. J. Exp. Med. 192:755-760.

5. Street, S.E., Trapani, J.A., MacGregor, D., and Smyth, M.J. 2002. Suppression of lymphoma and epithelial malignancies effected by interferon gamma. J. Exp. Med. 196:129-134.
6. Street, S.E., et al. 2004. Innate immune surveillance of spontaneous B cell lymphomas by natural killer cells and gammadelta T cells. J. Exp. Med. 199:879-884

7. Clementi, R., et al. 2005. A proportion of patients with lymphoma may harbor mutations of the perforin gene. Blood. 105:4424-4428.

8. Zerafa, N., et al. 2005. Cutting edge: TRAIL deficiency accelerates hematological malignancies. J. Immunol. 175:5586-5590. 
9. Davidson, W.F., Giese, T., and Fredrickson, T.N. 1998. Spontaneous development of plasmacytoid tumors in mice with defective Fas-Fas ligand interactions. J. Exp. Med. 187:1825-1838.

10. Airoldi, I., et al. 2005. Lack of Il12rb2 signaling predisposes to spontaneous autoimmunity and malignancy. Blood. 106:3846-3853.

11. Enzler, T., et al. 2003. Deficiencies of GM-CSF and interferon gamma link inflammation and cancer. J. Exp. Med. 197:1213-1219.

12. Coussens, L.M., and Werb, Z. 2002. Inflammation and cancer. Proc. Natl. Acad. Sci.U. S. A. 20:860-867.

13. Engel, A.M., Svane, I.M., Rygaard, J., and Werdelin, O. 1997. MCA sarcomas induced in scid mice are more immunogenic than MCA sarcomas induced in congenic, immunocompetent mice. Scand. J. Immunol. 45:463-470.

14. Svane, I.M., et al. 1996. Chemically induced sarcomas from nude mice are more immunogenic than similar sarcomas from congenic normal mice. Eur. J. Immunol. 26:1844-1850.

15. Girardi, M., et al. 2001. Regulation of cutaneous malignancy by gammadelta T cells. Science 294:605-609.

16. Smyth, M.J., et al. 2000. Differential tumor surveillance by natural killer (NK) and NKT cells. J. Exp. Med. 191:661-668.

17. Nishikawa, H., et al. 2005. Accelerated chemically induced tumor development mediated by CD4+CD25+ regulatory T cells in wild-type hosts. Proc. Natl. Acad. Sci. U. S. A. 102:9253-9257.

18. Crowe, N.Y., Smyth, M.J., and Godfrey, D.I. 2002. A critical role for natural killer $\mathrm{T}$ cells in immunosurveillance of methylcholanthrene-induced sarcomas. J. Exp. Med. 196:119-127.

19. van den Broek, M.E., et al. 1996. Decreased tumor surveillance in perforin-deficient mice. J. Exp. Med. 184:1781-1790.

20. Takeda, K., et al. 2002. Critical role for tumor necrosis factor-related apoptosis-inducing ligand in immune surveillance against tumor development. J. Exp. Med. 195:161-169.

21. Kaplan, D.H., et al. 1998. Demonstration of an interferon gamma-dependent tumor surveillance system in immunocompetent mice. Proc. Natl. Acad. Sci.U. S. A. 95:7556-7561.

22. Dunn, G.P., et al. 2005. A critical function for type I interferons in cancer immunoediting. Nat. Immunol. 6:722-729.

23. Smyth, M.J., et al. 2005. NKG2D function protects the host from tumor initiation. J. Exp. Med. 202:583-588.

24. Qin, Z., Kim, H.J., Hemme, J., and Blankenstein, T. 2002. Inhibition of methylcholanthrene-induced carcinogenesis by an interferon gamma receptor-dependent foreign body reaction. J. Exp. Med. 195:1479-1490.

25. Liu, J., Xiang, Z., and Ma, X. 2004. Role of IFN regulatory factor- 1 and IL-12 in immunological resistance to pathogenesis of $\mathrm{N}$-methyl-N-nitrosoureainduced T lymphoma. J. Immunol. 173:1184-1193.

26. Girardi, M., et al. 2003. The distinct contributions of murine $\mathrm{T}$ cell receptor (TCR)gammadelta+ and TCRalphabeta $+T$ cells to different stages of chemically induced skin cancer. J. Exp. Med. 198:747-755.

27. Oppenheim, D.E., et al. 2005. Sustained localized expression of ligand for the activating NKG2D receptor impairs natural cytotoxicity in vivo and reduces tumor immunosurveillance. Nat. Immunol. 6:928-937.

28. Langowski, J.L., et al. 2006. IL-23 promotes tumour incidence and growth. Proc. Natl. Acad. Sci. U. S. A 42:461-465.

29. Tartour, E., et al. 1999. Interleukin 17, a T-cellderived cytokine, promotes tumorigenicity of human cervical tumors in nude mice. Cancer Res. 59:3698-3704.

30. Numasaki, M., et al. 2003. Interleukin-17 pro- motes angiogenesis and tumor growth. Blood. 101:2620-2627.

31. Norbury, K.C., and Kripke, M.L. 1978. Ultraviolet carcinogenesis in T-cell-depleted mice. J. Natl. Cancer Inst. 61:917-921.

32. Ward, P.L., Koeppen, H.K., Hurteau, T., Rowley, D.A., and Schreiber, H. 1990. Major histocompatibility complex class I and unique antigen expression by murine tumors that escaped from CD8+ T-celldependent surveillance. Cancer Res. 50:3851-3858.

33. Mitra-Kaushik, S., Harding, J., Hess, J., Schreiber, R., and Ratner, L. 2004. Enhanced tumorigenesis in HTLV-1 tax-transgenic mice deficient in interferon-gamma. Blood. 104:3305-3311.

34. Willimsky, G., and Blankenstein, T. 2005. Sporadic immunogenic tumours avoid destruction by inducing T-cell tolerance. Proc. Natl. Acad. Sci. U. S. A. 37:141-146.

35. Ye, X., McCarrick, J., Jewett, L., and Knowles, B.B. 1994. Timely immunization subverts the development of peripheral nonresponsiveness and suppresses tumor development in simian virus 40 tumor antigen-transgenic mice. Proc. Natl. Acad. Sci.U. S. A. 91:3916-3920.

36. Buell, J.F., Gross, T.G., and Woodle, E.S. 2005. Malignancy after transplantation. Transplantation. 80:S254-S264.

37. Penn, I. 1988. Tumors of the immunocompromised patient. Annu. Rev. Med. 39:63-73.

38. Guidoboni, M., et al. 2001. Microsatellite instability and high content of activated cytotoxic lymphocytes identify colon cancer patients with a favorable prognosis. Am. J. Pathol. 159:297-304.

39. Buckowitz, A., et al. 2005. Microsatellite instability in colorectal cancer is associated with local lymphocyte infiltration and low frequency of distant metastases. Br. J. Cancer. 92:1746-1753.

40. Naito, Y., et al. 1998. CD8+ T cells infiltrated within cancer cell nests as a prognostic factor in human colorectal cancer. Cancer Res. 58:3491-3494.

41. Dolcetti, R., et al. 1999. High prevalence of activated intraepithelial cytotoxic $\mathrm{T}$ lymphocytes and increased neoplastic cell apoptosis in colorectal carcinomas with microsatellite instability. Am. J. Pathol. 154:1805-1813.

42. Ishikawa, T., et al. 2003. Tumor-specific immunological recognition of frameshift-mutated peptides in colon cancer with microsatellite instability. Cancer Res. 63:5564-5572.

43. Saeterdal, I., et al. 2001. Frameshift-mutationderived peptides as tumor-specific antigens in inherited and spontaneous colorectal cancer. Proc. Natl. Acad. Sci. U. S. A. 98:13255-13260.

44. Lu, B.J., Lai, M., Cheng, L., Xu, J.Y., and Huang, Q. 2004. Gastric medullary carcinoma, a distinct entity associated with microsatellite instability- $\mathrm{H}$, prominent intraepithelial lymphocytes and improved prognosis. Histopathology. 45:485-492.

45. Nakata, B., et al. 2002. Prognostic value of microsatellite instability in resectable pancreatic cancer. Clin. Cancer Res. 8:2536-2540.

46. Duval, A., et al. 2004. The mutator pathway is a feature of immunodeficiency-related lymphomas. Proc. Natl. Acad. Sci. U. S. A. 101:5002-5007.

47. Clark, W.H., Jr., et al. 1989. Model predicting survival in stage I melanoma based on tumor progression. J. Natl. Cancer Inst. 81:1893-1904.

48. Clemente, C.G., et al.1996. Prognostic value of tumor infiltrating lymphocytes in the vertical growth phase of primary cutaneous melanoma. Cancer 77:1303-1310.

49. Scanlan, M.J., Simpson, A.J., and Old, L.J. 2004. The cancer/testis genes: review, standardization, and commentary. Cancer Immun. 4:1

50. Haanen, J.B., et al. 2006. Melanoma-specific tumor-infiltrating lymphocytes but not circulating melanoma-specific T cells may predict survival in resected advanced-stage melanoma patients.
Cancer Immunol. Immunother. 55:451-458.

51. Dhodapkar, M.V. 2005. Immune response to premalignancy: insights from patients with monoclonal gammopathy. Ann. N. Y. Acad. Sci. 1062:22-28.

52. Kyle, R.A., et al. 2002. A long-term study of prognosis in monoclonal gammopathy of undetermined significance. N. Engl. J. Med. 346:564-569.

53. Dhodapkar, M.V., Krasovsky, J., Osman, K., and Geller, M.D. 2003. Vigorous premalignancy-specific effector $\mathrm{T}$ cell response in the bone marrow of patients with monoclonal gammopathy. J. Exp. Med. 198:1753-1757.

54. Gultekin, S.H., et al. 2000. Paraneoplastic limbic encephalitis: neurological symptoms, immunological findings and tumour association in 50 patients. Brain. 123:1481-1494.

55. Graus, F., et al. 1997. Anti-Hu antibodies in patients with small-cell lung cancer: association with complete response to therapy and improved survival. J. Clin. Oncol. 15:2866-2872.

56. Darnell, R.B., and DeAngelis, L.M. 1993. Regression of small-cell lung carcinoma in patients with paraneoplastic neuronal antibodies. Lancet. 341:21-22.

57. Tanaka, M., Tanaka, K., Tokiguchi, S., Shinozawa, K., and Tsuji, S. 1999. Cytotoxic T cells against a peptide of Yo protein in patients with paraneoplastic cerebellar degeneration and anti-Yo antibody. J. Neurol. Sci. 168:28-31.

58. Albert, M.L., et al. 1998. Tumor-specific killer cells in paraneoplastic cerebellar degeneration. Nat. Med. 4:1321-1324.

59. Mathew, R.M., Cohen, A.B., Galetta, S.L., Alavi, A., and Dalmau,J. 2006. Paraneoplastic cerebellar degeneration: Yo-expressing tumor revealed after a 5-year follow-up with FDG-PET. J. Neurol. Sci. 250:153-155.

60. Ferradini, L., et al. 1993. Analysis of T cell receptor variability in tumor-infiltrating lymphocytes from a human regressive melanoma. Evidence for in situ T cell clonal expansion. J. Clin. Invest. 91:1183-1190

61. Zorn, E., and Hercend, T. 1999. A natural cytotoxic $\mathrm{T}$ cell response in a spontaneously regressing human melanoma targets a neoantigen resulting from a somatic point mutation. Eur. J. Immunol. 29:592-601.

62. Zorn, E., and Hercend, T. 1999. A MAGE-6encoded peptide is recognized by expanded lymphocytes infiltrating a spontaneously regressing human primary melanoma lesion. Eur. J. Immunol. 29:602-607.

63. Halliday, G.M., Patel, A., Hunt, M.J., Tefany, F.J., and Barnetson, R.S. 1995. Spontaneous regression of human melanoma/nonmelanoma skin cancer: association with infiltrating CD4+ T cells. World J. Surg. 19:352-358.

64. Iihara, K., et al. 2004. Spontaneous regression of malignant lymphoma of the breast. Pathol. Int. 54:537-542.

65. Inoue, T., Yoneda, K., Manabe, M., and Demitsu, T. 2000. Spontaneous regression of Merkel cell carcinoma: a comparative study of TUNEL index and tumor-infiltrating lymphocytes between spontaneous regression and non-regression group. J. Dermatol. Sci. 24:203-211

66. Robinson, B.W., Robinson, C., and Lake, R.A. 2001. Localised spontaneous regression in mesothelioma - possible immunological mechanism. Lung Cancer. 32:197-201.

67. Kerr, K.M., et al. 1998. Partial regression in primary carcinoma of the lung: does it occur? Histopathology. 33:55-63.

68. Miura, K., Han, G., Sano, M., and Tsutsui, Y. 2002. Regression of congenital fibrosarcoma to hemangiomatous remnant with histological and genetic findings. Pathol. Int. 52:612-618.

69. Davis, T.A., Maloney, D.G., Czerwinski, D.K., Liles, T.M., and Levy, R. 1998. Anti-idiotype antibodies can induce long-term complete remissions in non- 
Hodgkin's lymphoma without eradicating the malignant clone. Blood. 92:1184-1190.

70. Montagna, D., et al. 2006. Emergence of anti-tumor cytolytic T cells is associated with maintenance of hematological remission in children with acute myeloid leukemia. Blood. 108:3843-3850.

71. Stewart, T.H., Hollinshead, A.C., and Raman, S. 1991. Tumour dormancy: initiation, maintenance and termination in animals and humans. Can. J. Surg. 34:321-325.

72. Callaway, M.P., and Briggs, J.C. 1989. The incidence of late recurrence (greater than 10 years); an analysis of 536 consecutive cases of cutaneous melanoma. Br. J. Plast. Surg. 42:46-49.

73. Demicheli, R., Abbattista, A., Miceli, R., Valagussa, P., and Bonadonna, G. 1996. Time distribution of the recurrence risk for breast cancer patients undergoing mastectomy: further support about the concept of tumor dormancy. Breast Cancer Res. Treat. 41:177-185.

74. Kauffman, H.M., McBride, M.A., and Delmonico, F.L. 2000. First report of the United Network for Organ Sharing Transplant Tumor Registry: donors with a history of cancer. Transplantation. 70:1747-1751.

75. Kauffman, H.M., et al. 2002. Transplant tumor registry: donor related malignancies. Transplantation. 74:358-362.

76. van Oijen, M., et al. 2004. On the role of melanomaspecific CD8+ T-cell immunity in disease progression of advanced-stage melanoma patients. Clin. Cancer Res. 10:4754-4760.

77. Kyewski, B., and Klein, L. 2006. A central role for central tolerance. Annu. Rev. Immunol. 24:571-606.

78. Schell, T.D., Knowles, B.B., and Tevethia, S.S. 2000 Sequential loss of cytotoxic T lymphocyte responses to simian virus 40 large $\mathrm{T}$ antigen epitopes in $\mathrm{T}$ antigen transgenic mice developing osteosarcomas. Cancer Res. 60:3002-3012.

79. van Elsas, A., Hurwitz, A.A., and Allison, J.P. 1999. Combination immunotherapy of B16 melanoma using anti-cytotoxic $\mathrm{T}$ lymphocyte-associated antigen 4 (CTLA-4) and granulocyte/macrophage colony-stimulating factor (GM-CSF)-producing vaccines induces rejection of subcutaneous and metastatic tumors accompanied by autoimmune depigmentation. J. Exp. Med. 190:355-366.

80. Park, J.M., et al. 2005. Early role of CD4+ Th1 cells and antibodies in HER-2 adenovirus vaccine protection against autochthonous mammary carcinomas. J. Immunol. 174:4228-4236.

81. Otahal, P., Schell, T.D., Hutchinson, S.C., Knowles, B.B., and Tevethia, S.S. 2006. Early immunization induces persistent tumor-infiltrating CD8+ $\mathrm{T}$ cells against an immunodominant epitope and promotes lifelong control of pancreatic tumor progression in SV40 tumor antigen transgenic mice. J. Immunol. 177:3089-3099.

82. Radoja, S., Rao, T.D., Hillman, D., and Frey, A.B. 2000 Mice bearing late-stage tumors have normal functional systemic $T$ cell responses in vitro and in vivo. J. Immunol. 164:2619-2628.

83. Kurt, R.A., et al. 1995. T lymphocytes infiltrating sites of tumor rejection and progression display identical $\mathrm{V}$ beta usage but different cytotoxic activities. J. Immunol. 154:3969-3974.

84. Kurt, R.A., Park, J.A., Schluter, S.F., Marchalonis, J.J., and Akporiaye, E.T. 2000. TCR v(beta) usage and clonality of $\mathrm{T}$ cells isolated from progressing and rejected tumor sites before and after in vitro culture. Int. Immunol. 12:639-646.

85. Blohm, U., et al. 2002. Lack of effector cell function and altered tetramer binding of tumor-infiltrating lymphocytes. J. Immunol. 169:5522-5530.

86. Zippelius, A., et al. 2004. Effector function of human tumor-specific CD8 $\mathrm{T}$ cells in melanoma lesions: a state of local functional tolerance. Cancer Res. 64:2865-2873.
87. Guilloux, Y., et al. 1994. Defective lymphokine production by most CD8+ and CD4+ tumor-specific $\mathrm{T}$ cell clones derived from human melanomainfiltrating lymphocytes in response to autologous tumor cells in vitro. Eur J. Immunol. 24:1966-1973.

88. Schwartz, R.H. 2003. T cell anergy. Annu. Rev. Immunol. 21:305-334.

89. Dong, H., et al. 2002. Tumor-associated B7-H1 promotes T-cell apoptosis: a potential mechanism of immune evasion. Nat. Med. 8:793-800.

90. Tripathi, P., and Agrawal, S. 2006. Non-classical HLA-G antigen and its role in the cancer progression. Cancer Invest. 24:178-186.

91. Derre, L., et al. 2006. Expression and release of HLA-E by melanoma cells and melanocytes: potential impact on the response of cytotoxic effector cells. J. Immunol. 177:3100-3107.

92. Campoli, M., Chang, C.C., and Ferrone, S. 2002. HLA class I antigen loss, tumor immune escape and immune selection [review]. Vaccine. 20(Suppl. 4):A40-A45.

93. Terabe, M., and Berzofsky, J.A. 2004. Immunoregulatory $\mathrm{T}$ cells in tumor immunity. Curr. Opin. Immunol. 16:157-162.

94. Zhang, Q., et al. 2005. Adoptive transfer of tumorreactive transforming growth factor-beta-insensitive CD8+ T cells: eradication of autologous mouse prostate cancer. Cancer Res. 65:1761-1769.

95. Chen, M.L., et al. 2005. Regulatory T cells suppress tumor-specific CD8 T cell cytotoxicity through TGF-beta signals in vivo. Proc. Natl. Acad. Sci. U. S. A. 102:419-424

96. Gorelik, L., and Flavell, R.A. 2001. Immune-mediated eradication of tumors through the blockade of transforming growth factor-beta signaling in T cells. Nat. Med. 7:1118-1122

97. Gabrilovich, D.I., Ishida, T., Nadaf, S., Ohm, J.E., and Carbone, D.P. 1999. Antibodies to vascular endothelial growth factor enhance the efficacy of cancer immunotherapy by improving endogenous dendritic cell function. Clin. Cancer Res. 5:2963-2970.

98. Kawamura, K., Bahar, R., Natsume, W., Sakiyama, S., and Tagawa, M. 2002. Secretion of interleukin10 from murine colon carcinoma cells suppresses systemic antitumor immunity and impairs protective immunity induced against the tumors. Cancer Gene Ther. 9:109-115.

99. McKallip, R., Li, R., and Ladisch, S. 1999. Tumor gangliosides inhibit the tumor-specific immune response. J. Immunol. 163:3718-3726.

100. Munn, D.H., and Mellor, A.L. 2007. Indoleamine 2,3dioxygenase and tumor-induced tolerance. J. Clin. Invest. 117:1147-1154. doi:10.1172/JCI31178.

101.Takahashi, H., et al. 2006. FAS death domain deletions and cellular FADD-like interleukin 1beta converting enzyme inhibitory protein (long) overexpression: alternative mechanisms for deregulating the extrinsic apoptotic pathway in diffuse large B-cell lymphoma subtypes. Clin. Cancer Res. 12:3265-3271.

102. Wohlfart, S., et al. 2004. FAS (CD95) mutations are rare in gastric MALT lymphoma but occur more frequently in primary gastric diffuse large B-cell lymphoma. Am. J. Pathol. 164:1081-1089.

103.Shin, M.S., et al. 2001. Mutations of tumor necrosis factor-related apoptosis-inducing ligand receptor 1 (TRAIL-R1) and receptor 2 (TRAIL-R2) genes in metastatic breast cancers. Cancer Res. 61:4942-4946.

104.Kataoka, T., et al. 1998. FLIP prevents apoptosis induced by death receptors but not by perforin/ granzyme B, chemotherapeutic drugs, and gamma irradiation. J. Immunol. 161:3936-3942.

105. Hinz, S., et al. 2000. Bcl-XL protects pancreatic adenocarcinoma cells against CD95- and TRAIL-receptor-mediated apoptosis. Oncogene. 19:5477-5486.

106.Serafini, P., Borrello, I, and Bronte, V. 2006. Myeloid suppressor cells in cancer: recruitment, phenotype, properties, and mechanisms of immune suppression. Semin. Cancer Biol. 16:53-65.

107. Kusmartsev, S., and Gabrilovich, D.I. 2006. Role of immature myeloid cells in mechanisms of immune evasion in cancer. Cancer Immunol. Immunother. 55:237-245.

108.Kusmartsev, S., et al. 2003. All-trans-retinoic acid eliminates immature myeloid cells from tumorbearing mice and improves the effect of vaccination. Cancer Res. 63:4441-4449.

109.Mirza, N., et al. 2006. All-trans-retinoic acid improves differentiation of myeloid cells and immune response in cancer patients. Cancer Res. 66:9299-9307.

110.Melani, C., Chiodoni, C., Forni, G., and Colombo, M.P. 2003. Myeloid cell expansion elicited by the progression of spontaneous mammary carcinomas in c-erbB-2 transgenic BALB/c mice suppresses immune reactivity. Blood. 102:2138-2145.

111.Song, X., et al. 2005. CD11b+/Gr-1+ immature myeloid cells mediate suppression of $\mathrm{T}$ cells in mice bearing tumors of IL-1beta-secreting cells. J. Immunol. 175:8200-8208.

112.Serafini, P., et al. 2004. High-dose granulocytemacrophage colony-stimulating factor-producing vaccines impair the immune response through the recruitment of myeloid suppressor cells. Cancer Res. 64:6337-6343.

113. Mazzoni,A.,etal. 2002. Myeloid suppressor lines inhibit $\mathrm{T}$ cell responses by an NO-dependent mechanism. J. Immunol. 168:689-695.

114. Bronte, V., et al. 2003. IL-4-induced arginase 1 suppresses alloreactive $\mathrm{T}$ cells in tumor-bearing mice. J. Immunol. 170:270-278.

115.Kusmartsev, S., Nefedova, Y., Yoder, D., and Gabrilovich, D.I. 2004. Antigen-specific inhibition of CD8+ T cell response by immature myeloid cells in cancer is mediated by reactive oxygen species. J. Immunol. 172:989-999.

116. Mantovani, A., Sozzani, S., Locati, M., Allavena, P., and Sica, A. 2002. Macrophage polarization: tumor-associated macrophages as a paradigm for polarized M2 mononuclear phagocytes. Trends Immunol. 23:549-555.

117.Smyth, M.J., Dunn, G.P., and Schreiber, R.D. 2006 Cancer immunosurveillance and immunoediting: the roles of immunity in suppressing tumor development and shaping tumor immunogenicity. Adv. Immunol. 90:1-50.

118.Bosma, G.C., Custer, R.P., and Bosma, M.J. 1983. A severe combined immunodeficiency mutation in the mouse. Nature. 301:527-530.

119.Hayashi, T., and Faustman, D.L. 2002. Development of spontaneous uterine tumors in low molecular mass polypeptide- 2 knockout mice. Cancer Res. 62:24-27.

120.Engel, A.M., et al. 1996. Methylcholanthreneinduced sarcomas in nude mice have short induction times and relatively low levels of surface MHC class I expression. APMIS. 104:629-639.

121.Street, S.E., Cretney, E., and Smyth, M.J. 2001. Perforin and interferon-gamma activities independently control tumor initiation, growth, and metastasis. Blood. 97:192-197.

122.Cretney, E., et al. 2002. Increased susceptibility to tumor initiation and metastasis in TNF-related apoptosis-inducing ligand-deficient mice.J. Immunol. 168:1356-1361.

123.Loser, K., et al. 2005. An important role of CD80/ CD86-CTLA-4 signaling during photocarcinogenesis in mice. J. Immunol. 174:5298-5305.

124.Johnsen, A.K. et al. 2001. Systemic deficits in transporter for antigen presentation (TAP)- 1 or proteasome subunit LMP2 have little or no effect on tumor incidence. Int. J. Cancer. 91:366-372.

125.Yue, H.H., Diehl, G.E., and Winoto, A. 2005. Loss of TRAIL-R does not affect thymic or intestinal tumor development in p53 and adenomatous polyposis coli mutant mice. Cell Death Differ. 12:94-97. 